\title{
A NEW OBJECTIVE PROCEDURE FOR THE ORTHOGONAL ROTATION IN FACTOR ANALYSIS (2)
}

\author{
SHIGEO KASHIWAGI \\ Fapanese National Railways
}

\begin{abstract}
One of the difficulties inherent in the normal varimax method of the orthogonal rotation of factors, i.e., the systematic bias caused by maximization of the sum of the variances of squared normalized factor loadings by columns, is pointed out. Then, it is shown that the method we have proposed in the preceding article (1963) provides a satisfactory solution of this difficulty by showing that the result of our solution, when applied on Thurstone's PMA battery, agrees well with his subjective solution.
\end{abstract}

\section{On the Systematic Bias by the Normal VARIMAX METHOD}

The determined rotation angle in the normal varimax method is obtained by maximization of the sum of the variances of squared normalized factor loadings by columns. In a certain kind of initial matrices, many negative large factor loadings result by doing this. This difficulty has already been pointed out by the present author (1963). Here, we are going to discuss another difficulty of this method.

Normalization of factor loadings was proposed by Kaiser (1958) in order to correct the systematic bias inherent in the raw varimax method which is caused by maximization of the sum of the variances of squared raw factor loadings by columns.

If almost all square roots of communalities of an initial factor matrix are one or nearly one, the normal varimax method is not different from the raw varimax method substantially. And, by normalization in two dimensional cases, we will find all factor loadings on the circumference of a circle with unit diameter and, in three dimensional cases, we will find them on the surface of a sphere with unit diameter. Generally, in $n$ dimensional cases we will find all factor loadings on the surface of a $n$ dimensional sphere with unit diameter. Then, it becomes apparent that the more the number of factors is, the less the effect of normalization will appear, because as the number of factors increases the greater number of single plane rotations are needed. The normal varimax solution is, in effect, the raw varimax solution of the normalized factor loadings. Therefore, it is apparent that the systematic bias in the raw varimax solution can not be corrected perfectly by the normal varimax method except for two dimensional cases. In the next section we will show how well our method (Kashiwagi, 1963) has solved this point.

\section{Results and Conclusions}

In Table 1 our solution of Harman and Holzinger's 8 physical variables (HarTABLE 1

Harman \& Holzinger's 8 physical variablest

\begin{tabular}{ccccccc}
\hline \multicolumn{1}{c}{ Varimax } & Solution & \multicolumn{3}{c}{ Subjective } & Our & Solution \\
Test & A & B & A & B & A & B \\
1 & 88 & 27 & 88 & 26 & 88 & 26 \\
2 & 92 & 21 & 92 & 20 & 92 & 20 \\
3 & 89 & 18 & 89 & 17 & 89 & 17 \\
4 & 86 & 25 & 86 & 24 & 86 & 23 \\
5 & 24 & 90 & 25 & 90 & 25 & 90 \\
6 & 18 & 79 & 19 & 79 & 20 & 79 \\
7 & 14 & 73 & 14 & 73 & 15 & 73 \\
8 & 25 & 68 & 26 & 68 & 26 & 68 \\
Vp & 332 & 265 & 335 & 261 & 336 & 260 \\
$\dagger$ & \multicolumn{7}{c}{ Decimal points omitted } & &
\end{tabular}




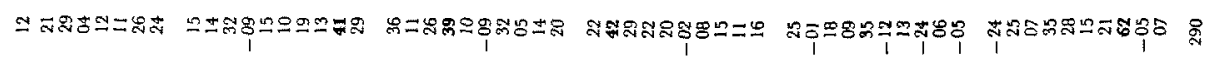

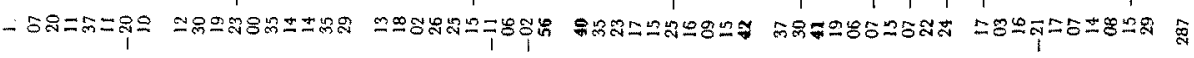

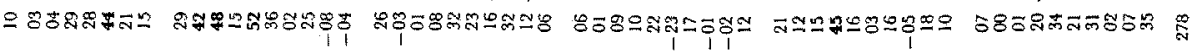

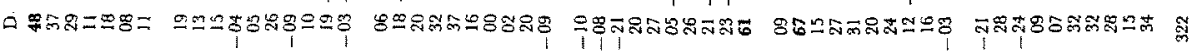

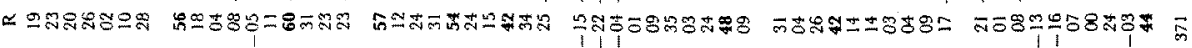

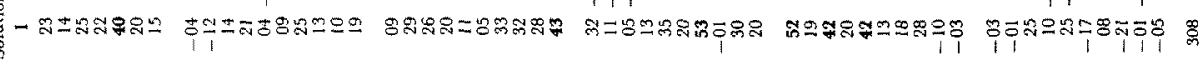

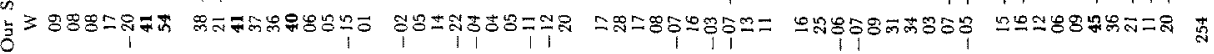

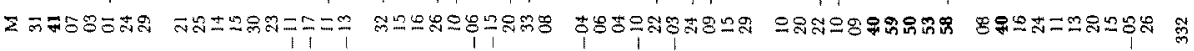

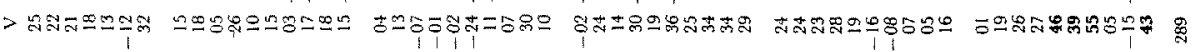

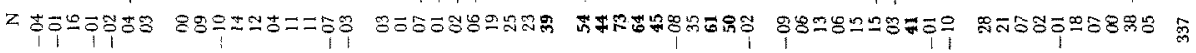

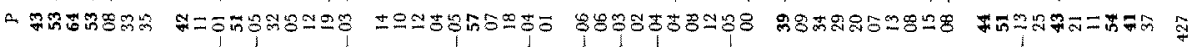

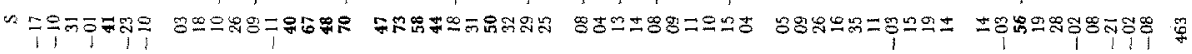

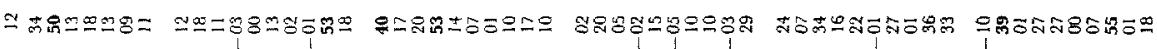

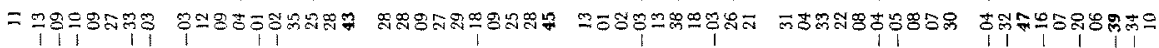

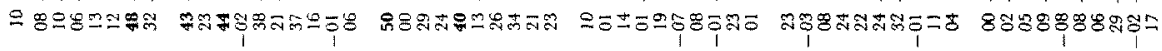

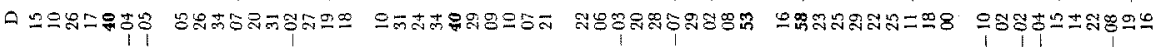

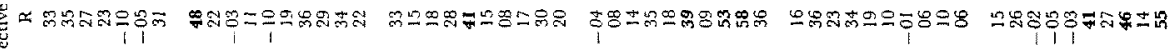

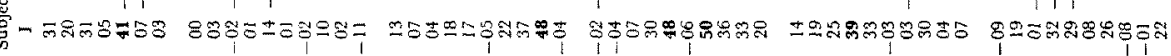
遂

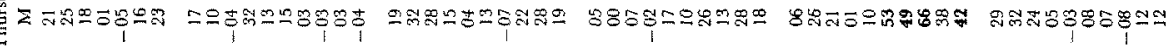

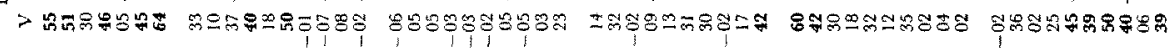

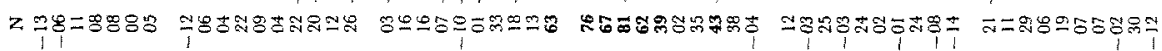

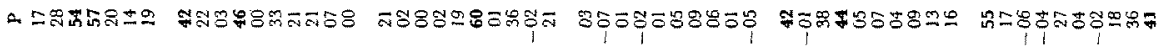

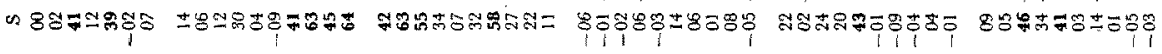

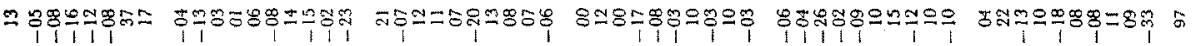

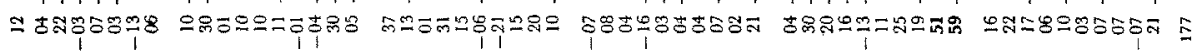

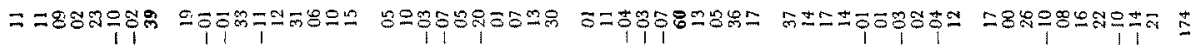

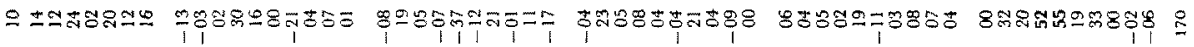

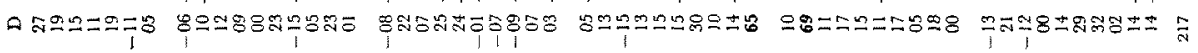

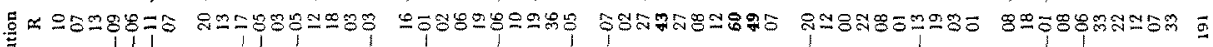

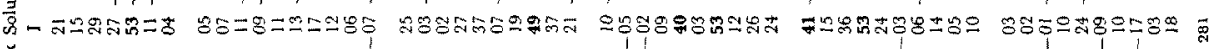
尊

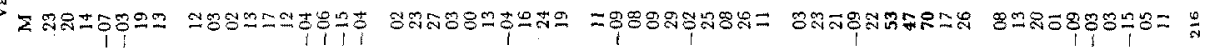

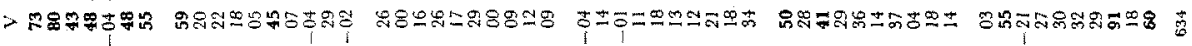
z

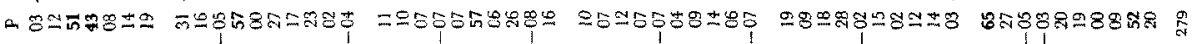
以

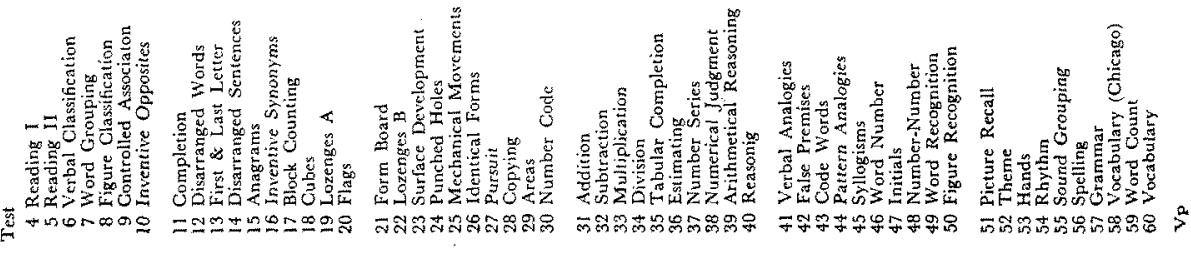


man, 1960) are shown together with the values which are determined by the normal varimax (Harman, 1960) and the subjective methods (Harman, 1960). In this example we can find how well our solution agrees with the subjective result. But, as its normal varimax solution which corrects for the systematic bias also agrees well with it, it is difficult to determine which result is closer to the subjective result. Nevertheless, if we compare our solution for Thurstone's Primary Mental Abilities of 12 centroid factors (1938) with its normal varimax solution of 13 centroid factors $(1960)^{1}$ in Table 2, the former is more appropriate than the latter in that it results in the better levelling of factor contributions (e.g., compare factor contributions of factor $\mathrm{S}$ and $\mathrm{V}$ in our solution with them in the normal varimax solution). And, if we take the subjective result (Thurstone, 1938) as the criterion, our solution agrees with it very well.
From these examples, we believe that our method satisfies most of Thurstone's criteria of the orthogonal rotation in factor analysis (e.g., positive manifold, simple structure and interpretability of factors).

\section{REFERENCES}

HaRman, H. H. Modern factor analysis. Chicago: Univ. of Chicago Press, 1960.

KAISER, H. F. The varimax criterion for analytic rotation in factor analysis. Psychometrika, 1958, 23, 187-200.

KAISER, H. F. Varimax solution for primary mental abilities. Psychometrika, 1960, 25, 153158.

Kashrwagi, S. A new objective procedure for the orthogonal rotation in factor analysis (1). fap. Psychol. Res. 1963, 5, 86-90.

Thurstone, L. L. Primary mental abilities. Psychometric Monogr. No. 1. Chicago: Univ. of Chicago Press, 1938.

(Received May 6, 1963)

1 It should be noted that Thurstone performed his subjective rotation with regard to 12 centroid factors but not with regard to 13 centroid factors. 